\title{
Liver Scan
}

National Cancer Institute

\section{Source}

National Cancer Institute. Liver Scan. NCI Thesaurus. Code C17647.

A radiologic procedure to examine the structure and/or function of the liver in which a small amount of a radiopharmaceutical is injected into the bloodstream to illuminate the area for the scanner. 